\section{AUSBLICK UND NÄCHSTE SCHRITTE}

Der Leitfaden hat zur Aufgabe, mögliche Wege für die Entwicklung von Forschung und Monitoring aufzuzeigen. Die Umsetzung hängt in von einer Anzahl sich wechselseitig beeinflussender Faktoren ab. Bei allem Bemühen zur Strukturierung und systematischen Weiterentwicklung des Themenfeldes „Forschung und Monitoring“ darf im Zuge der Umsetzung ein wenig Pragmatismus („Gelegenheiten am Schopf packen“, „Bewältigung lebenspraktischer Aufgaben ist der Theorie und Systematik dienenden Aufgabenerfüllung zumindest gleich zu stellen“) geübt werden. In anderen vergleichbaren Biosphärenparks hat sich gezeigt, dass Pragmatismus oft ein entscheidender Faktor für Weiterentwicklung ist.

\section{Stolpersteine}

Einige „Stolpersteine“ im Biosphärenpark Wienerwald verdienen besondere Beachtung, da sie großen Einfluss auf eine erfolgreiche Umsetzung nehmen:

- Prioritätenreihung: Der Biosphärenpark Wienerwald befindet sich in einer sehr dynamischen Entwicklungsphase. Man ist damit beschäftigt, funktionsfähige Grundstrukturen aufzubauen und Basiserhebungen durchzuführen. Forschung und Monitoring rücken in der Prioritätenliste an hintere Stelle.

- Personaleinsatz: Mit der Prioritätenreihung einher gehend wird die Wahrscheinlichkeit größer, dass eigentlich für Forschung und Monitoring vorgesehenes Personal anderwärtige Aufgabengebiete übernehmen muss.

- Budget: Ohne entsprechendes Basisbudget kann kaum Einfluss auf die Forschung genommen werden.

Vordringliche Aufgaben

- Einbindung der Forscherszene im Raum Wien: Der Leitfaden ist ein erster Schritt in Richtung Etablierung von Biosphärenparkforschung und zeigt die angestrebte
Entwicklungsrichtung (wohin wollen wir). Durch eine breit angelegte Internetbefragung wird im Februar/April die „science community“ zur Mitarbeit eingeladen. Über diesen Erstkontakt soll ein intensiver Austausch in Gang gesetzt werden und in absehbarer Zeit zu umsetzungsreifen Projekten und zum Aufbau eines Biosphärenparkforschungsnetzwerkes führen.

- Festlegung von Forschungsthemen: Eine vordringliche Aufgabe für das Biosphärenparkmanagement wird es sein, sich für gewisse Themenschwerpunkte, zu entscheiden, da diese Entscheidung Konsequenzen für nachfolgende Schritte mit sich bringt (z. B. Auswahl der Kooperationspartner). Mit der Erarbeitung einiger Projektsteckbriefe soll die „interne“ Entscheidung für Interessierte (Forscher, Studierende, Öffentlichkeit) sichtbar werden.

- Adaptierung an ein Rahmenkonzept: Forschung und Monitoring müssen in einen Gesamtrahmen gestellt werden, der vor allem durch von der strategischen Ausrichtung des Biosphärenparks (Vision, Leitbild, Ziele) bestimmt wird. Die Erstellung eines solchen Rahmenkonzeptes ist noch in Vorbereitung, wird aber jedenfalls mit den Zielsetzungen für Forschung und Monitoring abgestimmt.

Maßnahmenplan für die nächsten 2 Jahre

Die folgende Auflistung gibt einen groben Überblick über Maßnahmen, die in nächster Zeit gesetzt werden sollten, um den begonnenen Prozess möglichst rasch in die Umsetzungsphase überzuleiten.

\section{Kommunikation/Organisation}

- Einführung/Vorstellung des Konzepts und der nächsten Schritte im verantwortlichen Kreis

- Etablierung regelmäßiger Sitzungen zum Thema bzw. eines entsprechenden Tagesordnungspunktes bei den Biosphärenparksitzungen

- Treffen mit MaB-Nationalkomitee zur Abstimmung der weiteren Vorgangsweise (Kommunikationsstrategie, konkrete Hilfestellungen durch MaB-Komitee, Kooperation mit proVision etc.)

- Kontaktaufnahme mit möglichen Kooperationspartnern 
- Weiterführung der Forschungsfragensammlung

- Fixierung des Budgetrahmens

- Auswahl von Forschungsthemen und -fragen, die in ein konkretes Projekt münden sollen

- Einrichtung eines Forschungsbeirates

\section{Einführung von technischen Arbeitsinstrumenten}

- Erstellung einer Internetseite (allgemeine Informationen, Eingabe von Forschungsfragen, Forschungsschwerpunkte, aktuelle Projekte, Diplomarbeitsbörse)

- Einrichtung eines Metadatenkataloges

- Erstellung einer Projektdatenbank und Eingabe von ersten Projektsteckbriefen

- Einrichtung und Bewerbung einer Diplomarbeitsbörse auf der Homepage

- Etablierung einer Wissenschaftsseite in der Biosphärenparkzeitschrift 